\title{
Active behavior of abdominal wall muscles. Experimental results and numerical model formulation
}

\author{
J. Grasa ${ }^{a, c, *}$, M. Sierra ${ }^{\mathrm{a}}$, N. Lauzeral ${ }^{\mathrm{a}}$, M. J. Muñoz ${ }^{\mathrm{b}}$, F. J. Miana-Mena ${ }^{\mathrm{a}}$, B. \\ Calvo ${ }^{a, c}$ \\ ${ }^{a}$ Aragón Institute of Engineering Research. University of Zaragoza, Zaragoza, Spain. \\ ${ }^{b}$ Lagenbio, Aragón Institute of Engineering Research, University of Zaragoza, Zaragoza, \\ Spain \\ ${ }^{c}$ CIBER-BBN. Centro de Investigación en Red en Bioingeniería, Biomateriales y \\ Nanomedicina, Spain
}

\begin{abstract}
In the present study a computational finite element technique is proposed to simulate the mechanical response of muscles in the abdominal wall. This technique considers the active behavior of the tissue taking into account both collagen and muscle fiber directions. In an attempt to obtain the computational response as close as possible to real muscles, the parameters needed to adjust the mathematical formulation were determined from in vitro experimental tests. Experiments were conducted on male New Zealand White rabbits (2047 $\pm 34 \mathrm{~g})$ and the active properties of three different muscles: Rectus Abdominis, External Oblique and multi-layered samples formed by three muscles (External Oblique, Internal Oblique, and Transversus Abdominis) were characterized. The parameters obtained for each muscle were incorporated into a finite strain formulation to simulate active behavior of muscles incorporating the anisotropy of the tissue. The results show the potential of the model to predict the anisotropic behavior of the tissue associated to fibers and how this influences on the strain, stress and generated force during an isometric contraction.
\end{abstract}

Keywords: Abdominal muscle, in vitro active behavior, finite element method.

\footnotetext{
* Corresponding author

Email address: jgrasa@unizar.es (J. Grasa)
}

Preprint submitted to Journal of the Mechanical Behavior of Biomedical MaterialsMay 23, 2017 


\section{Introduction}

In mammals, the abdominal wall is composed of four muscle groups: Internal Oblique (IO), External Oblique (EO), Rectus Abdominis (RA) and Transversus Abdominis (TA). Unlike the thorax, internal organs are not protected by a bony structure and these muscles, together with fascial tissues, develop a protective function when acting passively. During active contractions, muscles in the abdominal wall participate in breathing, emesis, sneezing, coughing, defecation, micturition, phonation and postural control [1]. Anatomically, the IO lies internal to the EO muscle in the lateral abdominal wall, whereas the TA, the most internal abdominal muscle, lies in the lateral and ventral abdominal wall between the internal surface of the IO and the costal cartilage 2, 3. Each one of the previously quoted muscles has a specific muscle fiber orientation. The EO muscle fibers radiate caudally to the iliac crest and inguinal ligament and medially to the linea alba while the IO muscle fibers arise from the inguinal ligament and iliac crest and insert into the anterolateral surface of the cartilages of the last three ribs and into the linea alba, perpendicularly to the EO fibers. The TA muscle fibers run circumferentially around the abdominal visceral mass from the inner surface of the lower six ribs, lumbar fascia, iliac crest and the inguinal ligament to the rectus sheath are directed downward [4. Finally, the RA muscle fibers are parallel to the linea alba.

The active force developed by single muscle fibers is transmitted through a hierarchical structure of connective tissues to the muscle insertions or aponeuroses. The three anatomical parts of these connective tissues (from most external to most internal: epimysioum, perimysium and endomysium) are mostly woven collagen fibers embedded in an amorphous ground substance. In some

long strap-like muscles a two parallel sets of wavy collagen fibers in a crossed-ply arrangement have been observed in the epimysium [5. The collagen fibers are arranged at angles of approximately $55^{\circ}$ to the long axis of the muscle fibers. In other muscles, this arrangement is parallel to the muscle axis [5]. For the perimysium and endomysium, a distribution of collagen fibers running in all di- 
rections has been reported [6, 7, 5] covering bundles of muscle fibers and muscle fibers respectively. The three mentioned layers are connected together to transmit efficiently the muscle force. But focusing on the abdominal wall structure, another connective tissue plays an important role in the transmission of force and passive protection of internal organs that has become an object of increasing interest. This tissue, still without little consistent international terminology 8 is known as fascia and in the abdominal wall is located covering the muscles and between them $[3]$.

The unique anatomical arrangement of muscles and connective tissues in the abdominal wall has inspired descriptions and related hypotheses regarding its function as a composite-laminate structure [9, 10, 2]. Therefore, the material properties of this composite structure have been studied to better understand the abdominal wall mechanical behavior. The passive mechanical properties of the abdominal muscles have been investigated by several authors in different species: rat 2, 9, 10, rabbit 11, 12, pig [13, 14] and human [15. Moreover, the whole abdominal wall response to an increase of the intra-abdominal pressure has also been studied [16, 17, 18. Regarding the anisotropy of the tissue, this effect has been considered by [2] in a work where samples of tissue were loaded passively in two directions. Although these studies assist to understand how abdominal muscles behave, they focused only in the passive component meanwhile their active behavior remains unclear. Further research involving active behavior of this tissue would increase the knowledge to develop more and more efficient prosthesis in case of hernias meshes [3] or to understand the different contributions of muscles to the trunk stability [19, 20].

From a biomechanical point of view, muscle tissue presents some special characteristics as large deformations, anisotropic relationship between stress and applied strain and above all, a complex geometry. Consequently, closedform solutions of the mathematical equations cannot be found for non-trivial problems. The Finite Element Method (FEM) is a powerful tool to find good numerical solutions for these equations 21]. FEM has been successfully implemented for studying skeletal muscles with complex shapes for both active and 
passive behaviour $[22,23,24,25,26]$. The abdominal wall biomechanics has been studied by means of this technique assuming the presence of hernia defects 27. and the influence of different prostheses 28]. In these studies, only the passive behavior of the tissues was considered.

In the present study, the authors investigate the biomechanical characteristics of the abdominal wall contractions on New Zealand White rabbits. This animal model is commonly used for the study of hernia repair meshes [29, 30, 31. and authors characterized previously its passive response [3, 11]. In vitro experimental active tests are presented here for the RA, the EO and samples formed by three muscles (EO, IO and TA). Different parameters related to the active behavior were adjusted by means of a 3D electro-mechanical continuum model. This model, initially proposed by [32], has been modified here to take into account the influence of the fiber contraction velocity in the force development.

\section{Material and methods}

The experimental study was conducted on 10 male New Zealand White rabbits aged two months with a body mass of $2047 \pm 34 \mathrm{~g}$. All experiments were approved by the University of Zaragoza Ethics Committee for the use of animals in experimentation in accordance with the provisions of the European Council (ETS 123) and the European Union (Council Directive 86/609/EEC) regarding the protection of the animals used for experimental purposes. The animals were kept in a temperature controlled room $\left(22 \pm 1^{\circ} \mathrm{C}\right)$ with $12 \mathrm{~h}$ light-dark cycles and free access to water and food.

\subsection{In situ muscle preparation}

All animals were anesthetized by intramuscular injection of a mixture of Medetomidine $(0.14 \mathrm{mg} / \mathrm{Kg})$, Buprenorfine $(0.02 \mathrm{mg} / \mathrm{Kg})$ and Ketamine (20 $\mathrm{mg} / \mathrm{Kg}$ ) and euthanized by intravenous overdose of sodium pentobarbital. Immediately afterwards, animals were placed on their back and the abdominal skin was removed to define three different regions (Fig. 1). Two groups of animals 
were considered: in the first group $(n=5)$, samples of RA and EO were obtained for the first experiment described later; in the second group $(n=5) \mathrm{RA}$, EO and abdominal wall (EO-IO-TA) samples were used for the rest of experiments. For RA samples dissection, a $1 \times 3 \mathrm{~cm}$ rectangle was marked on the right side of the linea alba always above the umbilical area. For EO and abdominal wall samples, $3 \times 2 \mathrm{~cm}$ rectangles were delimited as can be observed in Fig. 1. Templates for the EO were oriented visually along muscle fiber directions. Within 2-5 min of death and for both groups, pieces of tissue were excised and "dogbone" samples were cut using a punch with a width/length ratio of 0.6 for the longitudinal direction. Each sample was attached, using cyanoacrylate cement, to sandpaper tabs to avoid slippage between the tissue and the clamps. The length, width and thickness of the samples were measured using a digital caliper.

In the RA excision, the rectus sheath was not separated from the underlying muscle to avoid tissue damage. A NIKON D5100 camera with a lens Tamrom $270 \mathrm{~mm}$ was used to observe the arrangement of muscle and collagen fibers in the external surface of a sample which is represented in Fig. 1. The internal surface is also represented in Fig. 1. The EO samples were excised approximately three centimeters at the left of the linea alba. The samples could be dissected easily separating the muscle from the underlying IO aponeuroses. The arrangement of muscle fibers could be seen with the naked eye (Fig. 1). Samples that include the three muscles of the abdominal wall were obtained from a region located five centimeters to the right from the linea alba.

\subsection{Protocol stimulation}

Each sample was oriented vertically in a methacrylate organ bath $(20 \times 20 \times 20$ $\mathrm{cm})$ showed in Fig. 2 and specifically designed by the authors to be installed in an electromechanical Instron Microtester 5248 with a $5 \mathrm{~N}$ full scale load cell. The lower end of the muscle was fixed inside the bath, where at the bottom of the container, a piece of plastic is screwed into a fixed one to configure a solid clamp. The upper end was fixed to the machine actuator by means of a grip. 
Temperature in the chamber is controlled pumping the bath solution (Ringer's solution) through a separate temperature controller and back to the organ bath. This system maintained the temperature at $27^{\circ} \mathrm{C}$ and the physiological solution was saturated with carbogen gas. A pair of platinum plate electrodes $(43 \times 30$ $\mathrm{mm}, 0.6 \mathrm{~mm}$ thickness) running the length of the sample was used to field stimulate the muscles in the different test protocols. Electrodes were connected to a CIBERTEC CS-20 electrical signal generator.

First experiment was carried out to find the muscle force-length relationship. Electrical pulses (1 ms duration and $100 \mathrm{~V}$ amplitude) were applied for the development of isometric twitch force varying muscle length. These different lengths subjected the muscle to several stretch levels $(\lambda=$ final length/initial length $)$ and were stablished taking decrements and increments of the initial sample length. The experimental protocol started always with the lower stretch values and after processing the results, the optimal stretch $(\lambda=1)$ was fixed at the point of maximum developed force. As described above, a first group of animals was used in this initial experiment to characterize the force-length relationship in the RA $(n=5)$ and in the EO $(n=5)$. The high levels of stretch applied to the samples inflicted damage that forced to discard them for the rest of the experiments. The muscle was rested for 2 min between successive stimuli at each length.

The second experiment was performed to determine the change generated on the force by increasing voltages. Samples of RA $(n=5)$ and EO $(n=5)$ were subjected to electrical pulses $(1 \mathrm{~ms}$ duration) from $40 \mathrm{~V}$ to $100 \mathrm{~V}$. The muscle length was fixed using the protocol in the previous experiment but with a reduced sweep of distances. Three to five different lengths were tested to find the optimum one ( 2 min resting intervals between stimuli). When the forcevoltage relationship was determined for each sample, the third experiment was performed using the same muscle after 5 min of rest. This experiment allowed to determine the force-frequency relationship applying train of pulses at increasing frequencies (from 10 to $100 \mathrm{~Hz}$ ) for $0.5 \mathrm{~s}$. Again, samples were rested for $2 \mathrm{~min}$ between stimuli. 
Samples of the abdominal wall $(n=5)$ were tested in the fourth experiment to determine its maximum isometric force. With this aim, They were oriented in the testing machine in the anatomical direction where fibers of the EO and IO where at $\pm 45^{\circ}$ and the TA fibers perpendicular to the longitudinal machine axis. Muscles were adjusted initially to the optimum length for isometric twitch force, they were stimulated with a single electrical pulse $(1 \mathrm{~ms})$ to produce a twitch response. Stimulation voltage was that which produced a maximal twitch response $(100 \mathrm{~V})$. Muscle stretch was adjusted very carefully in small increments (or decrements) to longer (or shorter) lengths (rest intervals of 2 min were taken). Optimal muscle stretch $(\lambda=1)$ was achieved when twitch force was maximal. At this optimal length three isometric tetanic contraction (0.5 s duration, $1 \mathrm{~ms}$ pulses at $100 \mathrm{~Hz}$ ) were applied resting 5 min.

\subsection{D hyperelastic constitutive laws}

The passive and active finite strain response of the muscle was simulated within the framework of continuum mechanics using a very common methodology based on postulating the existence of a strain energy function (SEF) [32, 33]. This function depends on the state variables $\mathbf{F}$ (deformation gradient), $\lambda_{a}$ (contraction or stretch of the muscle fibers) and of the structural tensors $\mathbf{M}=\mathbf{m}_{0} \otimes \mathbf{m}_{0}$ and $\mathbf{N}=\mathbf{n}_{0} \otimes \mathbf{n}_{0}$ which define the anisotropy of the muscle due to the preferential direction of muscular and collagen fibers, respectively. The function also depends on $\mathbf{C}_{e}$ which represents the elastic deformation of the cross-bridges.

$$
\Psi=\Psi\left(\mathbf{C}, \mathbf{C}_{e}, \lambda_{a}, \mathbf{N}, \mathbf{M}\right)
$$

The strain energy function is decoupled into a volume-changing and a volumepreserving parts in order to handle the quasi-incompressibility constraint. Furthermore, the deviatoric part is divided into a passive contribution, due to the collagen and elastin, $\bar{\Psi}_{p}$, and an active contribution associated with the muscular fibers, $\bar{\Psi}_{a}$. Thus, the total strain energy function $\Psi$ can be expressed as 
follows:

$$
\Psi=\Psi_{v o l}(J)+\bar{\Psi}_{p}(\overline{\mathbf{C}}, \mathbf{N})+\bar{\Psi}_{a}\left(\overline{\mathbf{C}}_{e}, \bar{\lambda}_{a}, \mathbf{M}\right)
$$

Following 32 Eq. 2 can be particularized for skeletal muscle and be formulated as:

$$
\Psi=\Psi_{\text {vol }}(J)+\bar{\Psi}_{p}\left(\bar{I}_{1}, \bar{I}_{2}, \bar{I}_{4}\right)+f_{\lambda} f_{V} f_{\text {train }} \bar{\Psi}_{a}^{\prime}\left(\bar{J}_{4}\right)
$$

The passive strain energy function, $\bar{\Psi}_{p}$, is defined as a function of the invariants:

$$
\bar{I}_{1}=\operatorname{tr} \overline{\mathbf{C}}, \quad \bar{I}_{2}=\frac{1}{2}\left((t r \overline{\mathbf{C}})^{2}-t r \overline{\mathbf{C}}^{2}\right), \quad \bar{I}_{4}=\mathbf{n}_{0} \cdot \overline{\mathbf{C}} \mathbf{n}_{0}=\bar{\lambda}^{2}
$$

where $\bar{I}_{1}$ and $\bar{I}_{2}$ are the first and second modified strain invariants of the symmetric modified Cauchy-Green tensor $\overline{\mathbf{C}}$, and $\bar{I}_{4}$ is the pseudo-invariant related to the anisotropy of the passive response (collagen fibers). According to the strain energy function proposed by [11], the passive response $\bar{\Psi}_{p}$ can be written:

$$
\bar{\Psi}_{p}=c_{1}\left(\bar{I}_{1}-3\right)+\frac{c_{3}}{c_{4}}\left(\exp ^{c_{4}\left(\bar{I}_{4}-\bar{I}_{4_{0}}\right)}-c_{4}\left(\bar{I}_{4}-\bar{I}_{4_{0}}\right)-1\right)
$$

The strain energy associated with the active response and consequently, with the actin-myosin interaction is expressed as the product of a series of functions that scale the maximum isometric stress able to generate the muscle. The active contribution of the strain energy function $\bar{\Psi}_{a}^{\prime}$ is expressed in terms of the pseudo-invariant associated to $\overline{\mathbf{C}}_{e}$ and the direction $\mathbf{m}_{0}[32$ :

$$
\bar{\Psi}_{a}^{\prime}=\frac{1}{2} P_{0}\left(\bar{J}_{4}-1\right)^{2} \quad \bar{J}_{4}=\mathbf{m}_{0} \cdot \overline{\mathbf{C}}_{e} \mathbf{m}_{0}=\bar{\lambda}_{e}^{2} .
$$

where $P_{0}$ is a proportionality factor related to the maximum active stress due to the muscle contraction 32. The influence of filament overlap on the active response of the muscle $f_{\lambda}$ is reformulated in terms of the muscle fiber stretch:

$$
f_{\lambda}=\exp \frac{\frac{-\left(\bar{\lambda}_{a}-\lambda_{o p t}\right)^{2}}{2 \xi^{2}}}{{ }^{2}}
$$


Here $\bar{\lambda}_{a}$ represents the deviatoric part of the muscle fiber stretch. $\lambda_{\text {opt }}$ defines the fiber stretch at which filaments overlap is optimum for force generation and $\xi$ adjusts the horizontal amplitude of the function. $f_{V}$ is expressed as:

$$
f_{V}=1-\exp ^{\frac{a-V}{d}}
$$

where $V$ is the voltage amplitude of the electrical stimulus, $a$ is the voltage value where no force response is obtained and $d$ controls the curvature of the function. The $f_{\text {train }}$ relationship is:

$$
f_{\text {train }}=\left(1-r e^{-f r \cdot c}\right) \sum_{i=1}^{n} P \frac{t-t_{\text {stim }}(i)}{T_{c}} e^{\left(1-\frac{\left(t-t_{\text {stim }}(i)\right)}{T_{c}}\right)}
$$

where $r$ and $c$ regulate the curvature of the initial and final slope of the force frequency relationship. $n$ is the number of stimulation pulses, $P$ and $T_{c}$ are the parameters defining twitch amplitude and apparent contraction time of the whole muscle, respectively, and $t_{s t i m}(i)$ is the time interval between stimulus $(i-1)$ and $i$.

According to [28] a constitutive relation must be satisfied to obtain the evolution of $\dot{\bar{\lambda}}_{a}$ :

$$
P_{a}-\frac{\partial \bar{\Psi}}{\partial \bar{\lambda}_{a}}+\left(2 \overline{\mathbf{C}}_{e} \frac{\partial \bar{\Psi}}{\partial \overline{\mathbf{C}}_{e}} \overline{\mathbf{F}}_{a}^{-T}\right): \frac{\partial \overline{\mathbf{F}}_{a}}{\partial \bar{\lambda}_{a}}=C \dot{\bar{\lambda}}_{a}
$$

In this relation, a new form of the active stress $P_{a}$ is proposed in this work:

$$
P_{a}=P_{0} f\left(\bar{\lambda}_{a}\right) f_{\text {train }} f\left(\dot{\bar{\lambda}}_{a}\right)
$$

This expression takes into account the dependence of the active force with respect to the contraction velocity. Thus, $f\left(\dot{\bar{\lambda}}_{a}\right)$ is assumed to be:

$$
f\left(\dot{\bar{\lambda}}_{a}\right)=\frac{1}{\nu} \dot{\bar{\lambda}}_{a}+1
$$

where $\nu$ represents the absolute value of the maximum concentric contraction 
velocity. A new parameter $C$ is also defined in the present work:

$$
C=\left(\frac{1}{v_{0}}\left(P-P_{0} f\left(\bar{\lambda}_{a}\right) f_{\text {train }}\right)\right)^{2}
$$

where $P$ is the total stress in the muscle fiber. Substituting Eqs. (11) and 13 in 10 , leads to the expression for $\dot{\bar{\lambda}}_{a}$ :

$$
\dot{\bar{\lambda}}_{a}=\frac{P_{0} f\left(\bar{\lambda}_{a}\right) f_{\text {train }}-\frac{\partial \bar{\Psi}}{\partial \bar{\lambda}_{a}}+\left(2 \overline{\mathbf{C}}_{e} \frac{\partial \bar{\Psi}}{\partial \overline{\mathbf{C}}_{e}} \overline{\mathbf{F}}_{a}^{-T}\right): \frac{\partial \overline{\mathbf{F}}_{a}}{\partial \bar{\lambda}_{a}}}{\left(\frac{1}{v_{0}}\left(P-P_{0} f\left(\bar{\lambda}_{a}\right) f_{\text {train }}\right)\right)^{2}-\frac{1}{\nu} P_{0} f\left(\bar{\lambda}_{a}\right) f_{\text {train }}}
$$

\subsection{Computational model}

A simplified model of a muscle sample was developed and meshed using ABAQUS software (Figure 3 a and 3 b). The chosen geometry for the EO and the RA is a 3D parallelepiped with the dimensions of the averaged sample measurements. In order to simulate the isometric contractions of the experimental tests, all the nodes of both ends of the model were fixed.

A second model was created combining both the EO, the IO and TA (Figure 3. c) in order to simulate the contraction of the oblique and transverse muscles contracting together. Three layers of muscles were disposed on the model with the same thickness of the experimental sample. In Figure 3 . c the direction of the muscle fibers in the three layers is represented. TA muscle fibers are disposed parallel to the $Y$ axis and the orientation for EO and IO are $45^{\circ}$ and $-45^{\circ}$ respectively with respect to the longitudinal dimension of the sample ( $X$ axis). The muscle layers were connected each other sharing the nodes in the interface regions and, as the previous models, all the nodes of both ends of the mesh were fixed.

Force developed by the models was measured as the reaction force in the fixed end regions. This force is actually the norm of the resultant of the force in every single fixed node. 


\section{Results}

\subsection{Experimental results}

The principal dimensions and weight of the samples are presented in Table 1. As can be observed, samples of the abdominal wall presented larger values of thickness and weight than RA and EO samples. As mentioned before, all the samples were cut using a punch so approximately, width dimensions are nearly the same.

The normalized force-stretch relationship, obtained for the EO and the RA is represented in Fig. 4. Forces developed in the experiment at the different stretch levels were normalized and then the mean and standard deviation were computed. The EO samples developed a maximum force of $0.215 \pm 0.019 \mathrm{~N}$ that represents a maximum isometric stress of $0.061 \pm 0.004 \mathrm{MPa}$. Maximum force for the RA samples was $0.351 \pm 0.088 \mathrm{~N}$ and $0.083 \pm 0.013 \mathrm{MPa}$ for the maximum isometric stress. The mean experimental results at each length were fitted using the Levenberg-Marquardt minimization algorithm and Eq. (7). The fitting for EO and RA force-stretch relationships are also presented in Fig. 4 The parameters in Eq. (7) and the results of the fitting are shown in Table 2

The experimental EO results obtained for the force-voltage relationship showed a maximum value at $100 \mathrm{~V}$ of $0.185 \pm 0.032 \mathrm{~N}(0.052 \pm 0.009 \mathrm{MPa})$. For RA samples, the maximum value of force registered at $100 \mathrm{~V}$ was $0.271 \pm 0.130$ $\mathrm{N}(0.064 \pm 0.012 \mathrm{MPa})$. This voltage was the maximum that the electrical stimulator generated. In the same way as the previous test, results were normalized and presented in Fig. 5 together with the fitting using Eq. 8 and the nonlinear least squares algorithm mentioned. Parameters $a$ and $d$ were determined for both samples (Table 2) with a coefficient of determination $R^{2}>0.94$. For both muscles, no significant force levels were observed under a signal amplitude of 40 V.

The experimental results obtained for the EO and RA samples in the forcefrequency relationship showed maximum force values starting from $70 \mathrm{~Hz}$. This force for the $\mathrm{EO}$ was $0.575 \pm 0.135 \mathrm{~N}(0.164 \pm 0.041 \mathrm{MPa})$. The RA samples 
developed $0.911 \pm 0.361 \mathrm{~N}$ starting at the same frequency level $(0.216 \pm 0.053$ $\mathrm{MPa}$ ). Parameters $r$ and $c$ in Eq. (9) were determined fitting the experimental results with a coefficient of determination $R^{2}>0.99$ (Table 2). Parameters $P$ and $T_{c}$ of the second product in Eq. (9) and $P_{0}$ in Eq. (6) were obtained fixing $f r=90 \mathrm{~Hz}$ and adjusting a 1D model (see [32]) to the mean response of samples in maximum isometric contractions.

For the abdominal wall samples, a maximum isometric force of $0.767 \pm 0.139$ $\mathrm{N}$ was observed when subjected to tetanic contractions. That represents a stress value of $0.051 \pm 0.01 \mathrm{MPa}$.

\subsection{Computational results}

Using the simplified computational models of the EO and RA samples, the last set of parameters in Table 2 related to the active part of the SEF was obtained. Following a similar procedure described in a previous work [34, a range of variation of $P_{0}, \nu_{0}$ and $\nu$ was defined and a large number of simulations was developed. The parameters were considered as uniformly distributed and the Latin hypercube sampling was used to generate a total of 1000 simulations. After post-processing those initial results the range of parameter variation was decreased and after a new set of 1000 simulations the best fit set of parameters is presented in Table 2 .

The reaction force in the computational model with the selected parameter combination is presented in Fig. 7 for the EO and RA muscles. In the same figure, the mean maximum force developed by the muscles in the experiment is also showed. This experimental force was that obtained for the force-frequency relationship at $100 \mathrm{~Hz}$ as described previously.

For simulating the isometric contraction of the multilayered abdominal wall muscle, the parameters obtained for the EO muscle were used for the IO and the TA. In Fig. 8. a the comparison between the computational force and the experimental one could be seen. The maximum force value developed by the model is $0.698 \mathrm{~N}$. Fig. 8 b represents the evolution of the muscle fibers active stretch $\left(\bar{\lambda}_{a}\right)$ and the active fiber contraction velocity $\left(\dot{\bar{\lambda}}_{a}\right)$ at the integration 
point of an element located in the central region of the mesh. The maximum stretch velocity reached by the model is $\dot{\bar{\lambda}}_{a}=-3.133 \mathrm{~s}^{-1}$ at the first increment and tends to $\dot{\bar{\lambda}}_{a}=0$ at the end of the contraction. The maximum active stretch was $\bar{\lambda}_{a}=0.4181$ at the end of the simulation.

Fig. 9 represents the evolution of the model contraction together with a color contour plot of the maximum principal stress. The maximum stress values (up to $1 \mathrm{MPa}$ ) are located at the ends of the mesh where the boundary conditions were defined. In the central region of the model the maximum principal stress is around $0.1 \mathrm{MPa}$ at the end of the contraction.

In Fig. 10 an exploded view has been used to represent the deformed configuration at the end of the contraction for the three muscle layers. In Fig. 10 the total stretch is plotted with a maximum elongation value of 1.663 located at one end of the EO muscle. The minimum shortening $(\bar{\lambda}=0.375)$ is located in the central region of the model.

Fig. 11. using an exploded view of the three muscle layers, shows muscle fiber directions in the deformed configuration using arrows. As can be observed in the central region of the model, fibers tend to align to guarantee a continuity in those directions between layers.

\section{Discussion and Conclusions}

The muscle fiber type composition establishes, among others properties, the maximum force developed and the contraction speed. In human abdominal muscles, there is a slow fiber (type I) predominance (50-60 \%) [35] and it has been described in the same way for the rabbit abdominal muscles: $60 \%$ fibers type I and $30 \%$ fibers type IIA in RA muscle [16. Slow fibers are characterized by low force values, slow reaction to the electrical stimulus, small size and fatigue resistance. These characteristics could explain the uniform response of both muscles (EO and RA) to the different voltage and frequency stimulus (Figs. 5 and 6). Comparing maximum force levels reached by the EO and RA, the latter developed a larger amount than the former due to its larger thickness. No 
significance differences were found between the maximum stress levels of both muscles when normalizing the maximum force by the cross sectional area. At this point, it is necessary to remark that all dimensions in Table1 1 were obtained after tissue extraction. When extracted, abdominal muscles exhibited a retraction that means a difference between the in vivo and the sample thicknesses. This fact has not been considered in the model due to the aim of validating only in vitro tests but in a more realistic model, this should be implemented in a similar way of previous works 24]. Furthermore, as mentioned in the text, the optimum length selected for the experiments was that of the maximum twitch force. This length has been recognized to be larger than the obtained for tetanic contractions [36. This fact could have resulted in an underestimation of the maximum force developed by the muscle samples. However, we chose this protocol in order to avoid muscle fatigue effects that could influence the load amplitude.

Parameters obtained by the fitting of the experimental results in the different relationships are quite similar for both the EO and RA (Table 2). This homogeneity is also observed in the parameters related to the active SEF that have been obtained by selecting the best approximation to the experimental mean curve of a model population 34. As can be observed in Figs. 7. a and 7. b the computational model fits the experimental behavior not only in the maximum contraction force but also in the shape of force evolution. The finite element mesh developed for the EO and RA muscles does not reproduce closely the real sample geometry in the region near the clamps of the machine. Although the cross sectional area in those regions of the real sample is affected, the results of the model should be interpreted carefully in the proximity of the boundary conditions.

A remarkable improvement of the previous active SEF [32] of the muscle model is presented in this work. The active stress is a function here of the active stretch velocity and the constitutive equation proposed for this velocity is a function of the stress in the muscle fiber. Despite this new definitions, the model maintains the important condition that is derived by applying fundamental 
principles in mechanics. Moreover, the expression of stretch velocity takes the form of the empirical Hill equation in a similar way as the work of [33. Fig. 8 represents the evolution of the state variable $\dot{\bar{\lambda}}_{a}$ of the model and the evolution of the active stretch in the fiber $\bar{\lambda}_{a}$. The velocity of the contraction is maximum at the beginning and during fiber orientation it diminishes until the equilibrium is achieved where the active stretch of the fiber remains constant.

The multi-layered muscle model (abdominal wall i.e. EO, IO and TA) was developed under the same considerations related to the geometry and boundary conditions mentioned previously for the EO and RA. In this model, the properties adopted for the IO and TA were the same as those determined for the EO. This assumption was motivated by the fact that extracting samples of these muscles avoiding fiber damage was extremely difficult. The homogeneity found in the properties of EO and RA made this assumption suitable and as can be observed in Fig. 8. a an error of less than $10 \%$ is obtained for the maximum force developed. In the same figure, the evolution of forces in both experimental and computational results in the first $0.2 \mathrm{~s}$ are very close. The gradual orientation of muscle fibers during muscle contraction observed in the model (Fig. 11) could explain the relative large time of the multi-layered muscle to reach the maximum contraction force. Comparing this time with those obtained experimentally in the mean curves for the one-layered muscles EO and RA, the time needed for the multi-layered sample is more than twice as long the one-layered samples. The model proposed for the three layer tissue assumes that there is not an interface between layers and that they are connected during contraction with no relative slide allowed. In a representation like the proposed in Fig. 10 the continuity of the stretch between layers could be observed. The anisotropy of the connective tissue in the interfaces has also not been considered. In all the models, directions $\mathbf{m}_{0}$ and $\mathbf{n}_{0}$ in Fig. 3 were taken to be the same, assuming that for isometric contractions, only connective tissue covering muscle fibers is involved. As can be observed in Fig. 10, elongation stretch values $(\bar{\lambda}>1)$ are achieved in regions near the model boundary conditions while in the rest of the model, shortening values $(\bar{\lambda}<1)$ are obtained in the direction of the fibers. This 
represents that the tissue is subjected mainly to a compressive state and only the volumetric and isotropic part of the SEF contribute to the passive behavior. Further analysis related to tissue behavior under compression should be considered. Although the results provided by the model are in good agreement with those observed experimentally, the mentioned limitation could be the reason why the model does not predict properly the maximum contraction force.

\section{Acknowledgements}

The authors gratefully acknowledge research support from the Spanish Ministry of Economy and Competitiveness (grants DPI2011-27939-C02-01 and DPI2014-54981-R), the University of Zaragoza (grant JIUZ-2014-BIO-05), Gobierno de Aragón and Fondo Social Europeo.

The authors also want to thank the Tissue Characterization Platform of CIBER-BBN for technical support during the experimental tests. CIBER-BBN is an initiative funded by the VI National R\&D\&i Plan 2008-2011, Iniciativa Ingenio 2010, Consolider Program, CIBER Actions and financed by the Instituto de Salud Carlos III with assistance from the European Regional Development Fund.

The work was performed by the ICTS "NANBIOSIS" specifically by the Tissue \& Scaffold Characterization Unit (U13), of the CIBER in Bioengineering, Biomaterials \& Nanomedicne (CIBER-BBN at the University of Zaragoza).

\section{References}

[1] M. Iizuka, Respiration-related control of abdominal motoneurons., Respir Physiol Neurobiol 179 (1) (2011) 80-88.

[2] W. Hwang, J. C. Carvalho, I. Tarlovsky, A. M. Boriek, Passive mechanics of canine internal abdominal muscles., J Appl Physiol (1985) 98 (5) (2005) $1829-1835$. 
[3] B. Hernández, E. Peña, G. Pascual, M. Rodríguez, B. Calvo, M. Doblaré, J. M. Bellón, Mechanical and histological characterization of the abdominal muscle. a previous step to modelling hernia surgery, J Mech Behav Biomed Mater 4 (3) (2011) 392-404.

[4] A. Ratnovsky, D. Elad, P. Halpern, Mechanics of respiratory muscles., Respir Physiol Neurobiol 163 (1-3) (2008) 82-89.

[5] P. P. Purslow, Muscle fascia and force transmission., J Bodyw Mov Ther 14 (4) (2010) 411-417.

[6] P. P. Purslow, The structure and functional significance of variations in the connective tissue within muscle., Comp Biochem Physiol A Mol Integr Physiol 133 (4) (2002) 947-966.

[7] B. R. MacIntosh, P. F. Gardiner, A. J. McComas, Skeletal muscle: form and function, Human Kinetics, 2006.

[8] C. Stecco, C. Tiengo, A. Stecco, A. Porzionato, V. Macchi, R. Stern, R. De Caro, Fascia redefined: anatomical features and technical relevance in fascial flap surgery., Surg Radiol Anat 35 (5) (2013) 369-376.

[9] S. H. Brown, J. M. Carr, S. R. Ward, R. L. Lieber, Passive mechanical properties of rat abdominal wall muscles suggest an important role of the extracellular connective tissue matrix., J Orthop Res 30 (8) (2012) 13211326.

[10] S. H. M. Brown, Mechanically relevant consequences of the composite laminate-like design of the abdominal wall muscles and connective tissues., Med Eng Phys 34 (4) (2012) 521-523.

[11] B. Calvo, M. Sierra, J. Grasa, M. J. Muñoz, E. Peña, Determination of passive viscoelastic response of the abdominal muscle and related constitutive modeling: stress-relaxation behavior, J Mech Behav Biomed Mater 36 (2014) 47-58. 
[12] R. Simón-Allué, J. M. M. Montiel, J. M. Bellón, B. Calvo, Developing a new methodology to characterize in vivo the passive mechanical behavior of abdominal wall on an animal model, J Mech Behav Biomed Mater 51 (2015) 40-9.

[13] M. Van Loocke, C. G. Lyons, C. K. Simms, Viscoelastic properties of passive skeletal muscle in compression: stress-relaxation behaviour and constitutive modelling, J Biomech 41 (7) (2008) 1555-66.

[14] M. Lyons, D. Winter, C. Simms, Mechanical characterisation of porcine rectus sheath under uniaxial and biaxial tension., J Biomech 47 (8) (2014) $1876-1884$.

[15] T. Förstemann, J. Trzewik, J. Holste, B. Batke, M. Konerding, T. Wolloscheck, C. Hartung, Forces and deformations of the abdominal wall-a mechanical and geometrical approach to the linea alba., J Biomech 44 (4) (2011) 600-606.

[16] E. V. Kotidis, T. S. Papavramidis, K. Ioannidis, A. Cheva, T. Lazou, N. Michalopoulos, G. Karkavelas, S. T. Papavramidis, The effect of chronically increased intra-abdominal pressure on rectus abdominis muscle histology an experimental study on rabbits., J Surg Res 171 (2) (2011) 609-614.

[17] W. M. Park, S. Wang, Y. H. Kim, K. B. Wood, J. A. Sim, G. Li, Effect of the intra-abdominal pressure and the center of segmental body mass on the lumbar spine mechanics - a computational parametric study., J Biomech Eng 134 (1) (2012) 011009.

[18] A. Rohlmann, L. Bauer, T. Zander, G. Bergmann, H. Wilke, Determination of trunk muscle forces for flexion and extension by using a validated finite element model of the lumbar spine and measured in vivo data., J Biomech 39 (6) (2006) 981-989.

[19] M. W. Olson, Comparison of trunk muscle reflex activation patterns be- 
tween active and passive trunk flexion-extension loading conditions., Hum Mov Sci 34 (2014) 12-27.

[20] J. P. Arokoski, T. Valta, O. Airaksinen, M. Kankaanpää, Back and abdominal muscle function during stabilization exercises, Arch Phys Med Rehabil 82 (8) (2001) 1089-98.

[21] C. W. J. Oomens, M. Maenhout, C. H. van Oijen, M. R. Drost, F. P. Baaijens, Finite element modelling of contracting skeletal muscle., Philos Trans R Soc Lond B Biol Sci 358 (1437) (2003) 1453-1460.

[22] M. Böl, S. Reese, Micromechanical modelling of skeletal muscles based on the finite element method, Comput Methods Biomech Biomed Engin 11 (5) (2008) 489-504. doi:10.1080/10255840701771750.

[23] C. Y. Tang, G. Zhang, C. P. Tsui, A 3d skeletal muscle model coupled with active contraction of muscle fibres and hyperelastic behaviour., J Biomech 42 (7) (2009) 865-872.

[24] J. Grasa, A. Ramírez, R. Osta, M. J. Muñoz, F. Soteras, B. Calvo, A 3d active-passive numerical skeletal muscle model incorporating initial tissue strains. validation with experimental results on rat tibialis anterior muscle., Biomech Model Mechanobiol 10 (5) (2011) 779-787.

[25] J. Grasa, M. Sierra, M. J. Muñoz, F. Soteras, R. Osta, B. Calvo, F. J. Miana-Mena, On simulating sustained isometric muscle fatigue: a phenomenological model considering different fiber metabolisms, Biomech Model Mechanobiol 13 (6) (2014) 1373-85.

[26] J. D. Webb, S. S. Blemker, S. L. Delp, 3d finite element models of shoulder muscles for computing lines of actions and moment arms, Comput Methods Biomech Biomed Engin 17 (8) (2014) 829-37. doi:10.1080/10255842. 2012.719605

[27] B. Hernández-Gascón, E. Peña, H. Melero, G. Pascual, M. Doblaré, M. P. Ginebra, J. M. Bellón, B. Calvo, Mechanical behaviour of synthetic surgical 
meshes: finite element simulation of the herniated abdominal wall., Acta Biomater 7 (11) (2011) 3905-3913.

[28] B. Hernández-Gascón, E. Peña, J. Grasa, G. Pascual, J. M. Bellón, B. Calvo, Mechanical response of the herniated human abdomen to the placement of different prostheses., J Biomech Eng 135 (5) (2013) 51004.

[29] A. Aramayo, F. Lopes, C. Barbosa, V. Amaral, L. Costa, Abdominal wall healing in incisional hernia using different biomaterials in rabbits., Acta Cir Bras 28 (4) (2013) 307-316.

[30] G. Pascual, S. Sotomayor, M. Rodríguez, Y. Bayon, J. M. Bellón, Behaviour of a new composite mesh for the repair of full-thickness abdominal wall defects in a rabbit model, PLoS One 8 (11) (2013) e80647.

[31] E. Peeters, K. van Barneveld, M. Schreinemacher, G. De Hertogh, Y. Ozog, N. Bouvy, M. Miserez, One-year outcome of biological and synthetic bioabsorbable meshes for augmentation of large abdominal wall defects in a rabbit model., J Surg Res 180 (2) (2013) 274-283.

[32] B. Hernández-Gascón, J. Grasa, B. Calvo, J. F. Rodríguez, A 3D electromechanical continuum model for simulating skeletal muscle contraction, J Theor Biol 335 (2013) 108-18.

[33] B. Sharifimajd, J. Stålhand, A continuum model for skeletal muscle contraction at homogeneous finite deformations, Biomech Model Mechanobiol 12 (5) (2013) 965-73.

[34] M. Sierra, F. J. Miana-Mena, B. Calvo, M. J. Muñoz, J. F. Rodríguez, J. Grasa, On using model populations to determine mechanical properties of skeletal muscle. application to concentric contraction simulation, Ann Biomed Eng 43 (10) (2015) 2444-55.

[35] S. Iscoe, Control of abdominal muscles., Prog Neurobiol 56 (4) (1998) 433506. 
[36] D. E. Rassier, B. R. MacIntosh, Length-dependent twitch contractile characteristics of skeletal muscle, Can J Physiol Pharmacol 80 (10) (2002) 9931000. 


\begin{tabular}{|c|c|c|c|}
\hline & RA & Abdominal wall & EO muscle \\
\hline Weight $(\mathrm{g})$ & $0.222 \pm 0.022$ & $0.910 \pm 0.014$ & $0.168 \pm 0.059$ \\
\hline Length $(\mathrm{mm})$ & $23.64 \pm 4.20$ & $38.00 \pm 3.32$ & $29.79 \pm 6.98$ \\
\hline Width $(\mathrm{mm})$ & $5.12 \pm 0.22$ & $5.14 \pm 0.24$ & $5.21 \pm 0.24$ \\
\hline Thickness $(\mathrm{mm})$ & $0.82 \pm 0.20$ & $2.90 \pm 0.12$ & $0.67 \pm 0.14$ \\
\hline
\end{tabular}

Table 1: Average dimensions of studied samples (mean \pm standard deviation) 


\begin{tabular}{ccc}
\hline & EO & RA \\
\hline \hline Force-Stretch relationship & & \\
$\lambda_{\text {opt }}$ & 1 & 1 \\
$\xi$ & 0.1383 & 0.1814 \\
$R^{2}$ & 0.9469 & 0.9866 \\
\hline
\end{tabular}

Force-Voltage relationship

\begin{tabular}{ccc}
$a(\mathrm{~V})$ & 14.1800 & 6.0130 \\
$d(\mathrm{~V})$ & 28.6800 & 24.0900 \\
$R^{2}$ & 0.9617 & 0.9471 \\
\hline
\end{tabular}

Force Frequency relationship

$\begin{array}{ccc}r & 0.9968 & 0.9846 \\ c & 0.0670 & 0.0761 \\ R^{2} & 0.9957 & 0.9919\end{array}$

Force Time relationship

\begin{tabular}{rcc}
$f r(\mathrm{~Hz})$ & 90 & 90 \\
$P(\mathrm{~N})$ & 0.0221 & 0.0196 \\
$T_{c}(\mathrm{~s})$ & 0.04 & 0.04 \\
\hline Active SEF & & \\
$P_{0}(\mathrm{MPa})$ & 0.0463 & 0.0734 \\
$v_{0}$ & 17.411 & 14.4831 \\
$\nu\left(\mathrm{s}^{-1}\right)$ & 3.1658 & 2.2649 \\
\hline
\end{tabular}

Table 2: Parameters determined from the experimental tests to fit the different relationships for the EO and RA muscles. 


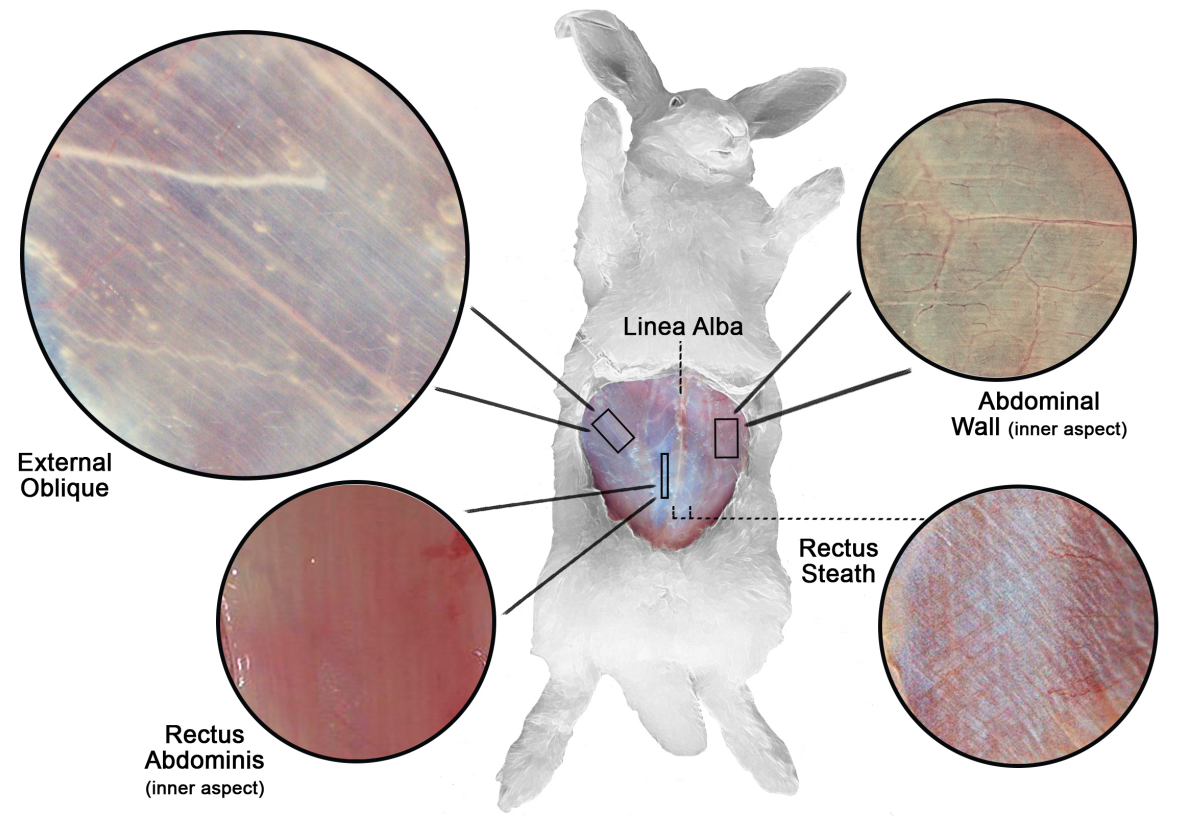

Figure 1: Scheme of the dissection regions and main orientations of muscle and collagen fibers in the samples. 


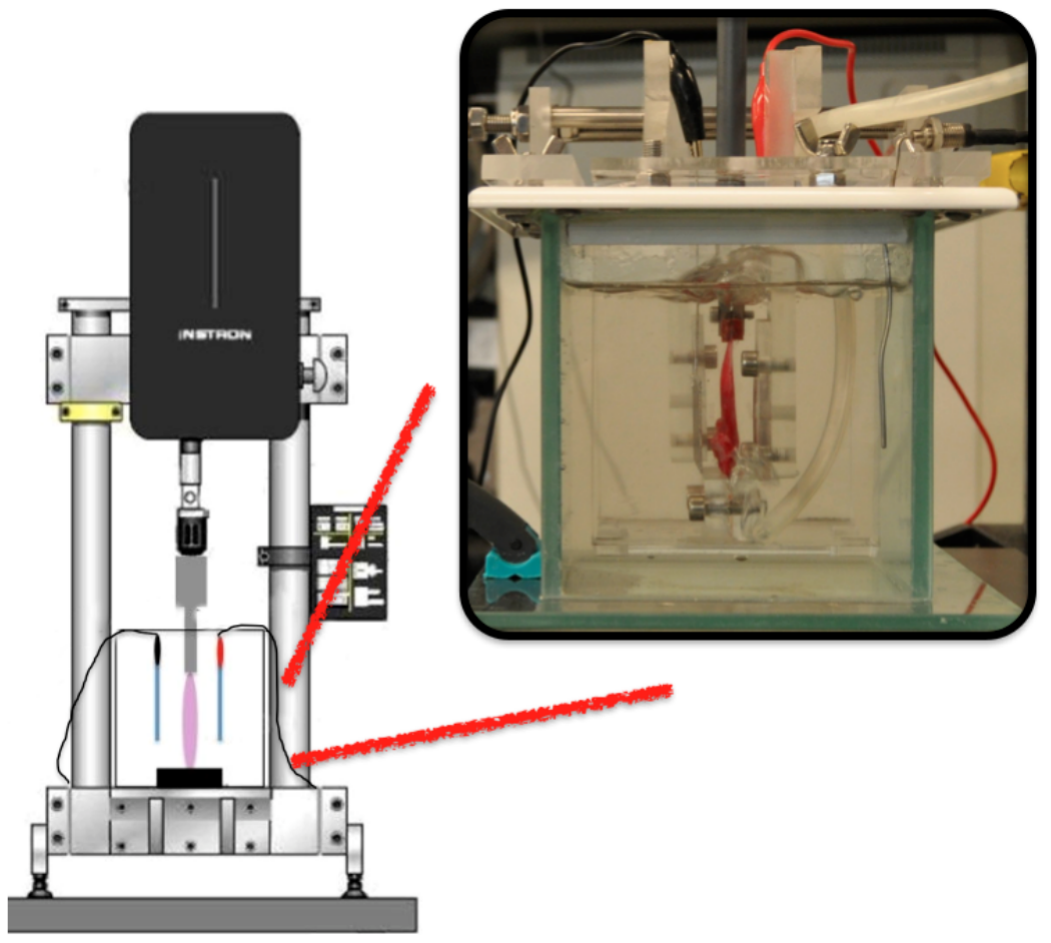

Figure 2: Scheme of the experimental setup. A methacrylate organ bath is placed in an universal electromechanical testing machine (Instron Microtester 5248) where the muscle is fixed and stimulated by means of two platinum plates. 


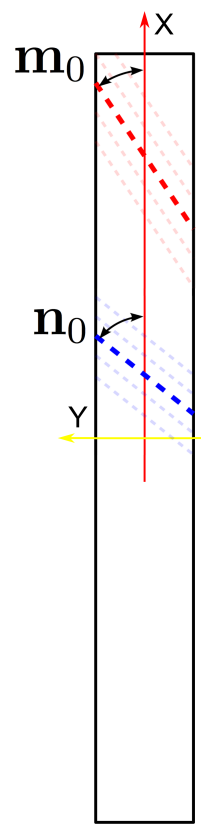

(a)

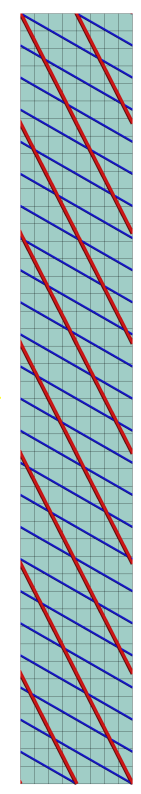

(b)

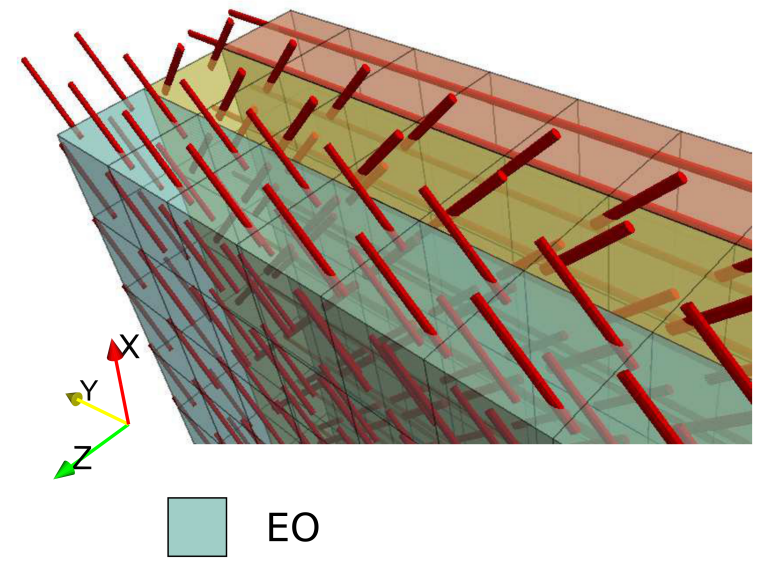

10

TA

Figure 3: Anisotropic behavior definition in the computational model. (a) The direction $\mathbf{n}_{0}$ is associated with the preferential orientation of collagen fibers and the direction $\mathbf{m}_{0}$ with the orientation of muscle fibers. (b) Schematic representation of fibers in the simplified model of the experimental sample. (c) Schematic representation of the muscle fibers in the simplified model of the abdominal wall. 


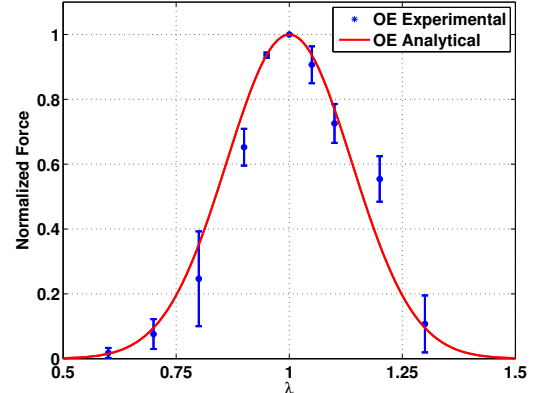

(a)

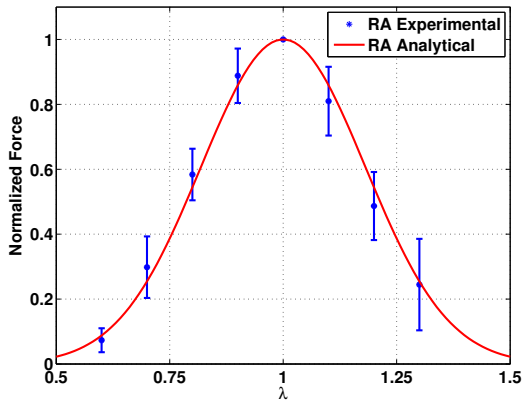

(b)

Figure 4: Force-Stretch relationship (a) EO relationship (normalized by EO maximum force) (b) RA relationship (normalized by RA maximum force). 


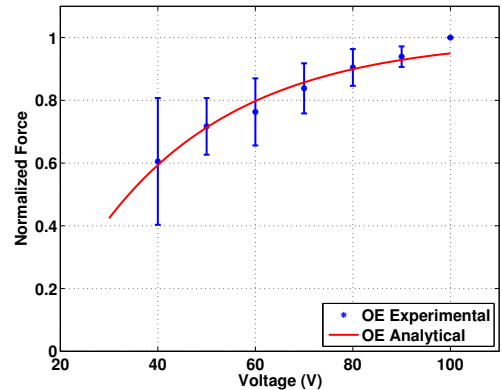

(a)

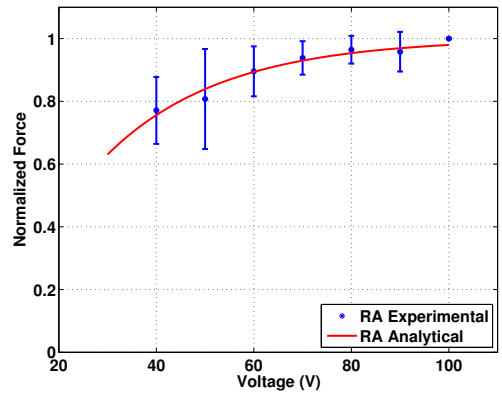

(b)

Figure 5: Force-Voltage relationship (a) EO relationship (normalized by EO maximum force) (b) RA relationship (normalized by RA maximum force). 


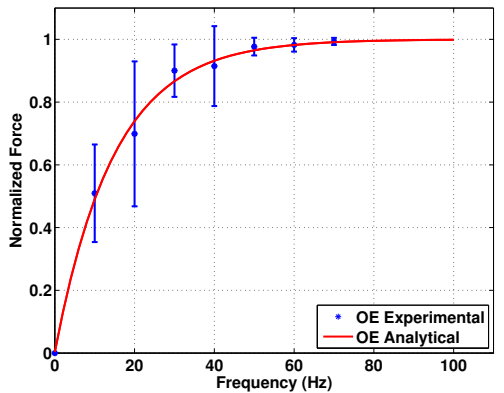

(a)

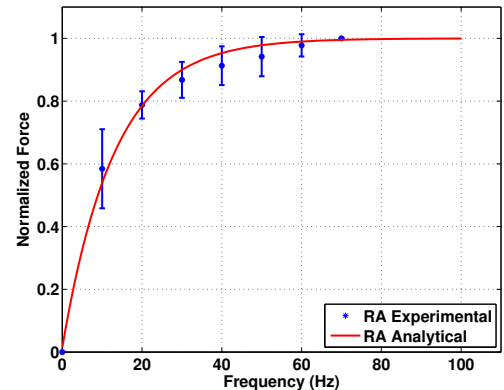

(b)

Figure 6: Normalized force-frequency relationship, experimental results and model fitting for (a) EO and (b) RA. 


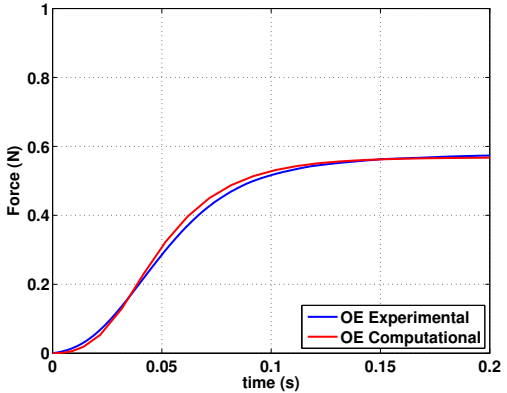

(a)

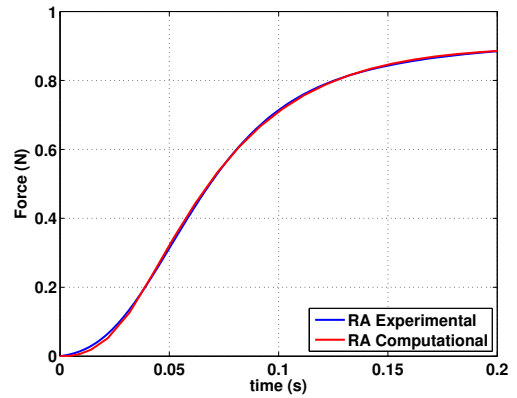

(b)

Figure 7: Mean maximum force response of experimental samples fitted by the three dimensional computational model for (a) EO and (b) RA muscles. 


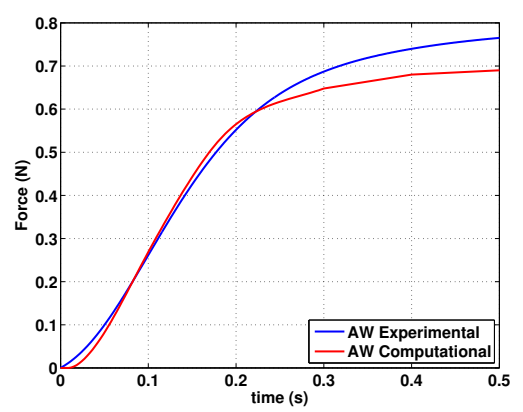

(a)

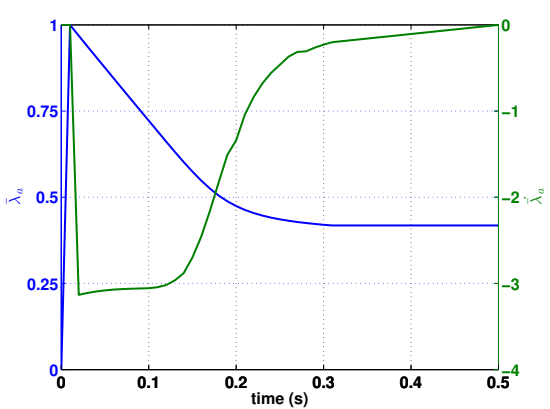

(b)

Figure 8: (a) Comparison between mean experimental contraction force of the multilayered abdominal wall muscle and the computational model. (b) Evolution of $\bar{\lambda}_{a}$ and $\dot{\bar{\lambda}}_{a}$ at the integration point of an element in the central region of the model. 


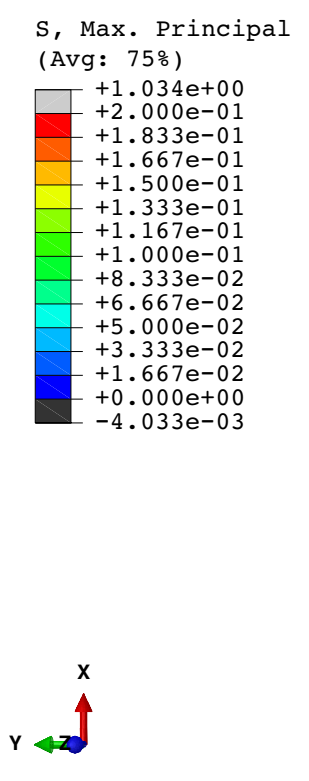

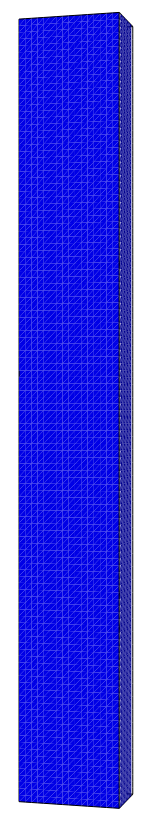

a

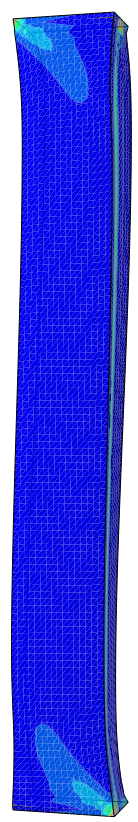

b

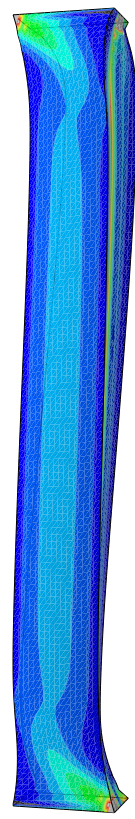

C

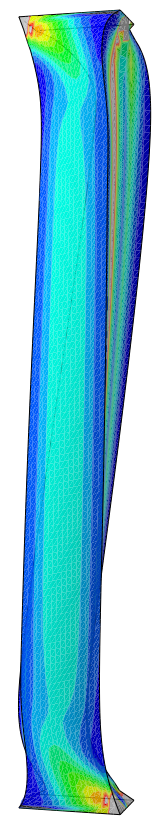

d

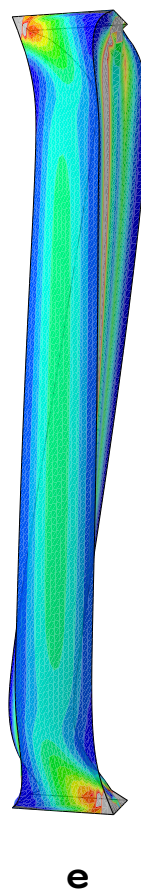

e

Figure 9: Maximum principal stress in the computational model of the three muscle layers at: (a) $0 \mathrm{~s}$, (b) 0.05 , (c) 0.1, (d) 0.2, (e) 0.3 . 


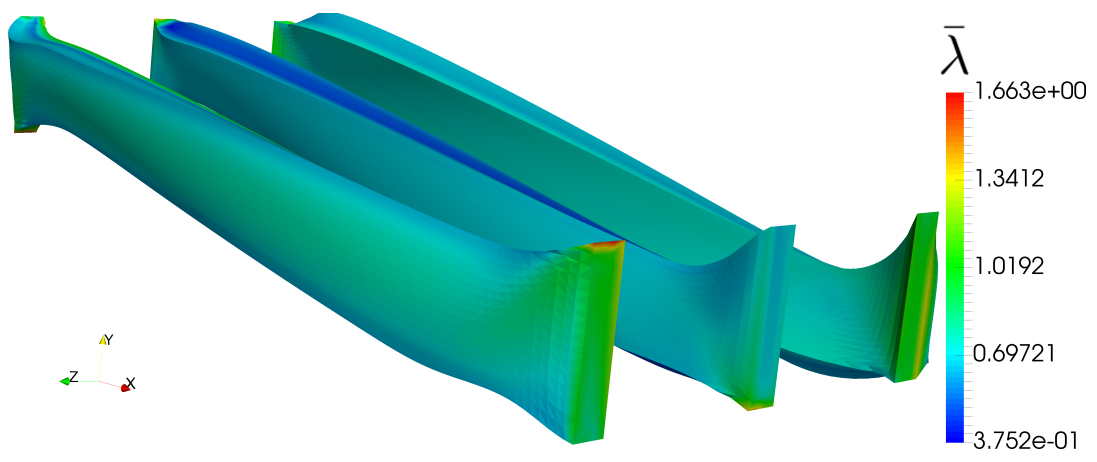

Figure 10: Exploded view of the three muscle layers, from left to right EO, IO and TA. The variable represented in the colour contours is the total stretch evaluated at the mesh nodes $(t=0.5 \mathrm{~s})$. 


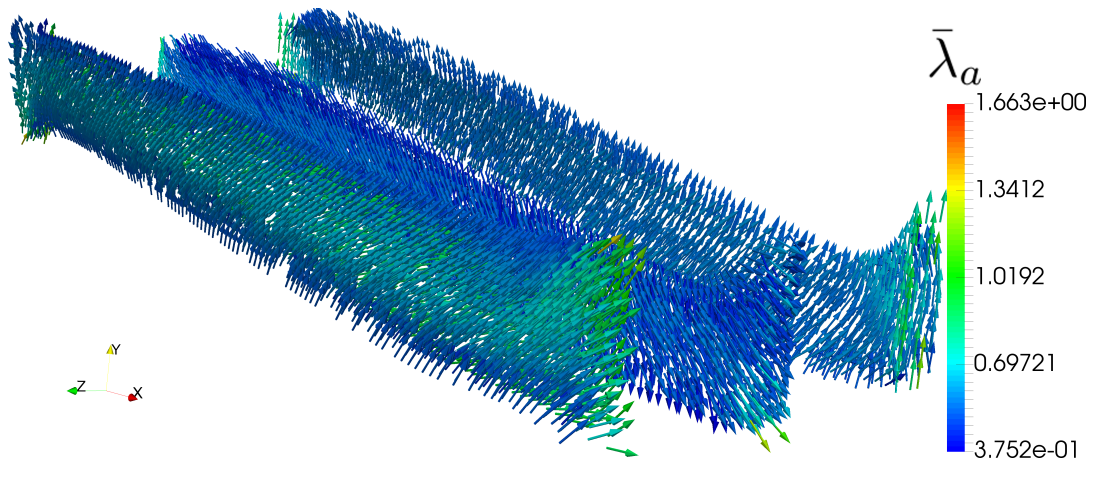

Figure 11: Exploded view of the three muscle layers, from left to right EO, IO and TA. Arrows represent the muscle fiber directions in the deformed configuration. The variable represented in the color contours is the active stretch evaluated at the mesh nodes $(t=0.5 \mathrm{~s})$. 